\title{
Lebensgefährliche Bradykardie durch Chinincocktail
}

Eine ältere Dame hatte noch einmal Glück - fast wäre sie einer Arrhythmie erlegen: Hydroxychloroquin gegen Rheuma, Chinin gegen Wadenkrämpfe, dazu ein Gin Tonic. Da reichte eine kleine Virusinfektion für den Kollaps.

Chininhaltige Arzneien werden eigentlich kaum noch medizinisch angewandt, in den USA sind sie sogar nur noch gegen Malaria zugelassen. Das hält aber manche Patienten und auch manchen Arzt nicht davon ab, weiterhin auf die segensreiche Wirkung des ChinarindenbaumAlkaloids zu setzen. Dabei ist längst bekannt, dass Chinin auch problematische Nebeneffekte haben kann, die gerade für ältere Patienten relevant sind. So blockiert das Alkaloid Natriumkanäle, verzögert dadurch die Depolarisierung und begünstigt damit eine QT-Zeitverlängerung. Diese wurde einer 91-jährigen Frau aus New Mexico fast zum Verhängnis.

\section{Torsadogene Tachykardie-Episoden} Wie Internisten um Dr. Elyce Sheehan von der Uniklinik in Albuquerque berichten, wurde die Frau in einem delirösen Zustand in die Klinik eingeliefert. Familienangehörige hatten die Patientin in ihrem Stuhl und Erbrochenen vorgefunden. In der Klinik angekommen, war sie sehr verwirrt und agitiert, daher sedierten sie die Ärzte.

Das initiale EKG zeigte episodenhaft eine nicht anhaltende polymorphe ventrikuläre Tachykardie bei einem Puls von 50/min. Die Ärzte in der Notaufnahme setzten die Patientin auf Amiodaron und überstellten sie in die Intensivstation. Das EKG ergab dort in der ersten Nacht eine ausgeprägte Sinusbradykardie und eine massiv verlängerte QT-Zeit von mehr als 800 Millisekunden. Immer wieder traten auch torsadogene Episoden einer nicht anhaltenden polymorphen ventrikulären Tachykardie auf, die sich auf die QT-Zeitverlängerung zurückführen ließen.

Die Ärzte fanden zudem spezifische ventrikuläre Extrasystolen, die einer ventrikulären, Torsades-de-Pointes-artigen
Tachykardie vorausgingen. Sie setzen daraufhin Amiodaron ab und verwendeten stattdessen Lidocain als potenziellen QTZeit-Verkürzer.

Im Laufe der nächsten Tage normalisierte sich die QT-Zeit wieder und die Patientin erholte sich fast vollständig.

\section{"Chininismus" durch Gin Tonic}

Als sie wieder klar im Kopf war, wollte sie auch sogleich ihre Wadenkrampfmedizin. Dies lehnten die Ärzte freundlich, aber bestimmt ab. Wie sich inzwischen herausgestellt hatte, war ihr vom Hausarzt Chinin gegen die Krämpfe verordnet worden, gegen ihr Rheuma hatte sie Hydroxychloroquin genommen, und $\mathrm{zu}$ allem Überfluss gönnte sie sich abends regelmäßig einen Gin Tonic. Auslöser für den Kollaps war aber wohl eine Magen- darmgrippe, die über Appetit- und Flüssigkeitsmangel die Chinintoxizität noch verstärkt hatte.

Dass Chinin die QTZeit verlängern kann, war bereits in den 1930er-Jahren aufgefallen. Ein Arzt des Gouverneurs Jim Folsom aus Alabama erkannte diese Veränderung im EKG seines Patienten. Dieser hatte über anhaltenden Tinnitus geklagt. Zunächst ging der Arzt beim EKG noch von einem Zufallsbefund aus, riet dem Politiker jedoch, seinen exzessiven Konsum von Gin Tonic einzustellen, da er einen "Chininismus" vermutete. Folsom wechselte zu Bourbon - und die Symptome verschwanden.

Thomas Müller

Sheehan ET et al. Quinine and the ABCs of Long QT: A Patient's Misfortune with Arthritis, (Alcoholic) Beverages, and Cramps. J Gen InternMed 31(10):1254-7

\section{Clostridium-difficile-Infektion}

\section{Säurehemmer erhöhen das Rezidivrisiko bei CDI}

Patienten mit Clostridium-difficileInfektion (CDI) haben ohnehin ein hohes Rezidivrisiko. Die Anwendung von Medikamenten, die die Magensäuresekretion hemmen, scheint es zusätzlich zu steigern.

In einer amerikanischen Metaanalyse zum Einfluss der Säuresuppression auf Rezidive von CDI wurden 16 Beobachtungsstudien mit zusammen 7703 CDIPatienten berücksichtigt. Von ihnen hatten $20 \%$ innerhalb von 90 Tagen ein CDIRezidiv durchgemacht. Patienten mit PPI- und/oder H2-Blocker-Therapie waren signifikant häufiger davon betroffen; die Rezidivrate betrug 22\% im Vergleich zu $17 \%$ bei Patienten ohne eine solche Medikation. Damit war das Rezidivrisiko unter Säurehemmern um 52\% erhöht.

Für die Zunahme von CDI unter Säurehemmern werden sowohl der Verlust der schützenden Magensäure als auch Veränderungen im Darmmikrobiom verantwortlich gemacht.

Raseen Tet al. JAMA Intern Med 2017, online 27. März DOl:10.1001/jamainternmed.2017.0212 